\title{
An Open Flow Controller Based on the Intercerebral Neural Network for Media Independent Handover
}

\author{
Qiong Wu, Jun S Huang ${ }^{*}$ and Oliver WW Yang
}

3rd Department, Suqian College, Jiangsu 223800, P.R. China

*Corresponding author: Dr. Jun S. Huang, 3rd Department, Suqian College, Jiangsu 223800, P.R. China, Tel: +86 52784203000 ; Fax: +86 52784202300 ; E-mail: steedhuang@ujs.edu.cn

Received date: March 15, 2014, Accepted date: August 23, 2014, Published date: August 30, 2014

Copyright: $\odot 2014$ Wu Q, et al. This is an open-access article distributed under the terms of the Creative Commons Attribution License, which permits unrestricted use, distribution, and reproduction in any medium, provided the original author and source are credited.

\begin{abstract}
This paper presents a novel open flow controller based on the intercerebral neural network for the media independent handover over wireless operation. This controller makes a binary decision based on the link control parameters obtained instantaneously from the trained neural network, which in turn depends on the instantaneous mobile speed interaction with the wireless link performance parameters. These parameters of packet loss, round trip time, radio signal strength, bandwidth, data cost and vehicle speed are typically different from network to network. We use a mutated particle swarm optimization to train the fundamental controller equation for the media independent handover that are mixed with the radial and sigmoid activation functions of the intercerebral neural network. Simulations based on the field experiment test data show that the system using the swarm intelligence algorithm is practical for the improvement of the overall secure networking experience for the fast delivery.
\end{abstract}

Keywords: Handover; Neural; Radial; Sigmoid; Swarm

\section{Introduction}

A transportation company for fast delivery service usually has to maintain a team of service trucks on the road, each of which is equipped with a mobile scanner that keeps track of the goods during its upload and download operations as well as reporting the GPS position of the truck. The dispatching schedule and the route of each truck are semi-fixed because the route is not fixed like a bus but also not purely random like a tax driver's either. For each truck driver, the schedule and route are dictated by a handful of fixed customers, which are typically optimized by the so-called swan goose algorithm [1] to save the total amount of gasoline needed for the day. The challenges remain to maximize the throughput of the uploading the logistical information, while the dispatching truck is going through the semifixed route (relatively fixed route on the roughly fixed schedule).

Currently either the WiFi-based mobile scanner [2] or the GPRSbased mobile scanner are used to support the communication of logistical information which includes pictures of 1-D bar codes or 2-D QR codes, as well as sensitive credit card information, where no breach of information is allowed. WiFi-based operation is fast, but only available within limited areas. Although GPRS-based is more accessible, it is fairly expensive, especially in dealing with large amount of bulky pictures. There is also a security problem when a cheap broken link may allow the attacker to easily obtain information on payment transaction. To minimize this risk; we need a solid and smart algorithm to continuously monitor the overall quality of each network. To handle the economical dilemma, we also need to further improve the performance of the system of scanners by combining the access methods of WiFi and GPRS. We would like to design a smart mobile handover algorithm using the latest evolutional computation algorithm to maximize the secured throughput but with the minimum handover cost between the WiFi and the GPRS media. To achieve this, we look to the implementation using SDN (Software Defined Network).

Started as an approach to computer networking [3] a few years ago, SDN allows network administrators to manage network services through the abstraction of lower level functionalities. A communication system in general has two planes: a control plane that can make decision where traffic is sent, and the data plane that can forward traffic to the selected destination. SDN decouples the control plane in a system from the data plane in the underlying systems. In addition to the simplification of networking issues, we believe it can also lower the overall cost as to be shown in this paper.

There are different communication methods between the control plane and the data plane. We would like to use the Open Flow method from the Open Networking Foundation (ONF) [4], which also markets the use of "cloud computing" before it became popular. Dynamic resource allocation is one important aspect of an elastic Cloud architecture. As the usage of mobile computer operating systems grows, such as the growing number of scanners used in the booming dispatching industry, the need has arisen for an additional layer in SDN. This layer should allow network operators to specify network services (e.g., uploading or downloading express dispatching information and sensitive credit card code) without coupling these specifications with network interfaces (e.g. interfaces to $\mathrm{WiFi}$, WiMAX, GPRS or LTE). This enables entities to move between interfaces without changing identities or violating secured service specifications. It can also simplify network operations, where global definitions per identity do not have to be matched to each and every interface location when a service truck is driving through the entire city or among cities. Such a layer can also reduce some complexity built up in network elements; this is achieved by decoupling the identity and flow-specific control logic from the basic topology-based operations of forwarding, bridging, and routing on top of the wireless handover. 
The global software defined control also tracks down specific flow contexts based on source and destination identity aspects. A mechanism for driving network hardware has been added and adopted by network equipment manufacturers for the purpose of sharing edge driving between software-defined edge and vendor-specific bridging and routing. A set of open commands for forwarding was defined in the form of a protocol known as Open Flow. The Open Flow protocol enables globally-aware software controllers, which may be centralized or distributed, to drive the network edge hardware in order to create an easily programmable identity-based overlay on top of the traditional IP core.

The wireless use case enables Open Flow to effectively perform both break-before-make and make-before-break flow assignments by using Media Independent Handover (MIH) protocol commands [5] as specified by IEEE802.21, which is under development within IEEE802 group. It is expected that 802.21 is well suited for Open Flow implementation, because Open Flow is specified in a media independent manner.

Our study further employs the particle swarm training algorithm to optimize the media independent solution. This can be extended to more new networks, such as LTE or WiMAX. There was a demonstration of the previous application of PSO for Computer Numerically Controlled (CNC) machine tool design [6,7], which inspired our new finding that is driven by our industrial sponsor Jufeng Group in Danyang Development Zone, who is dedicated to transportation and storage logistics.

\section{Need for an Intercelebral Neural Network for Mobile Handover Controller}

To minimize the data cost consumed during the dispatching operation, both the WiFi system and GPRS system have to be seamlessly handover; such that the summation of both providing the maximum and secure throughput. As the truck starts or stops there will be a WiFi connection to the office, as the truck moves on the road, there will be GPRS service along the road. Note that during the normal driving period, the GPS data is small, while during the start or stop time, where picture and credit card signature are collected, the data is relatively large and sensitive; we thus need the IEEE 802.21 compatible Open Flow controller to switch between the two networks.

Typical wireless handover is determined by looking at the Link Going Down (LGD) parameter [8], LGD can be a function of a number of measured parameters, such as packet loss, round trip time, radio signal strength, bandwidth, data cost and vehicle speed, etc. Each of these parameters has a relationship to the location environment relative to the base station or access point. Take the most used parameter, Radio Signal Strength Indicator (RSSI) as an example, the relationship is not linear with respect to the transmitter to receiver distance; and the typical RSSI value for WiFi and GPRS are also different. As such, when we trying to combine all parameters into one decision parameter, it becomes highly complicated nonlinear, and slightly random, as the schedule and route can be deviated from the daily routing, the best way to learn this kind of the function is through special class of the neural network, which we call it Intercerebral Neural Network (INN).

An intercerebral neuron network structure is proposed instead of one pure kind, for the following reason: Each measured parameters has its own underline nonlinear functions, which are stemmed from completely different roots, by using the exact the same neuron to describe them is hard. Note that the switch from WiFi to GPRS will be different from switch GPRS to WiFi at least. A typical dispatching system is shown in Figure 1. The car A is driving from GPRS 4+1 WiFi into $802.11 \mathrm{~b}$, and car B is driving from $802.11 \mathrm{~b}$ to WiFi $802.11 \mathrm{n}$, car C is from WiFi $802.11 \mathrm{n}$ to GPRS $3+2$, so on so forth. We need to train the neuron in such a way, that the decision is optimum for all the potential cases.

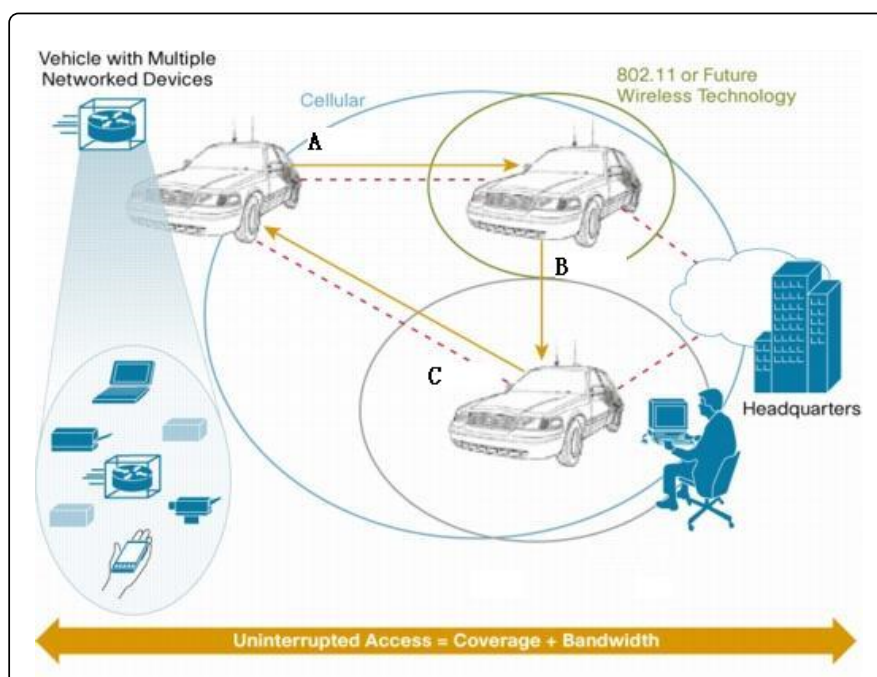

Figure 1: Wireless handover model.

It is important to note that the controller makes the decision by using a Particle Swarm Optimization (PSO) [9] trained intercerebral neural network on the current measured parameters. By monitoring the current parameters, we can automatically balancing the cost with trained system. The algorithm learns how to increase the secured throughput without causing too much extra charges, based on the collected fields' data.

Due to the semi fixed schedule and route, there is the need to enhance previous MIH methods [10] for the following reasons:

- The direct calculation model cannot follow the route variation from the extra customer calls occurred on the road.

- The complicated adaptive model method can only partially deal with the route variation, but does not treat the schedule changes very well.

- The homogeneous Neural Network (NN) can work, but we found that the parameters trained for one truck does not fit the other.

The method that can handle the above four considerations is the INN trained with a mutated PSO method [11]. The intercerebral approach comes from studying of actual neuron structure between man and woman, where we found that there are only two types of neurons in our brain; we call it either RBF $[12,13]$ or SBF:

a) A Radial Basis Function (RBF) network [14] is a neural network that uses radial basis functions as activation functions. The output of the network is a linear combination of radial basis functions of the inputs and neuron parameters. Radial basis function networks have many uses, including function approximation, time series prediction, classification, and system control. 
b) A Sigmoid Basis Function (SBF) is a mathematical function having an "S" shape (sigmoid curve). Often, sigmoid function refers to the special case of the logistic function. Another example is the Gompertz curve. It is used in modeling systems that saturate at large values of input, e.g. the ogee curve as used in the spillway of some dams $[15,16]$. A wide variety of sigmoid functions have been used as the activation function of neurons, including the logistic and hyperbolic tangent functions. Sigmoid curves are also common in statistics as cumulative distribution functions, such as the integrals of the logistic distribution, the normal distribution, and Student's $t$ probability density functions.

c) According to the biological concept, RBF is related to brain memory function, more on our left brain, and SBF is related to brain imagination capability, more on right brain. The structure of RBF supports the academic school of connectivitilism [17], while that of SBF favors the computational believers. In our opinion, SBF offers nonlinear effects for large input value; RBF provides nonlinear effect at small input value. A combination of both SBF and RBF will result in more nonlinear blending across the entire region. We use the mix of both to learn the input and output relations with multiple nonlinear root causes.

d) Psychological studying shown that woman can learn and adapt to new social environment faster than man. A recent study used diffusion MRI to map functional differences [18] in male and female brains. In the cerebral cortex it was observed that, in comparison with the opposite sex, there was greater intracelebral neural communication in male brains and greater intercerebral neural communication in female brains. In another word, woman may have more blending of nonlinear functions in the intercelebrum for them to plasticity the neurons to new culture environment faster. This finding leads us to pay attention to the intercerebral neuron network. The training of such mixed network becomes a bit challenging, for this reason, we picked the mixed PSO method with part of Genetic Algorithm (GA).

PSO is a computational method that optimizes a problem by iteratively trying to improve a candidate solution with regard to a given measure of quality. PSO optimizes a problem by having a population of candidate solutions, here dubbed particles, and moving these particles around in the search-space according to simple mathematical formulae over the particle's position and velocity. Each particle represents a combination of network conditions, say Monday to Friday, good or bad, GPRS or WiFi, that's $5 \times 2 X 2=20$ in total. Each particle's movement is influenced by its local best known position, but is also guided toward the best known positions in the search-space, which are updated as better positions are found by other particles. This is expected to move the swarm toward the best solutions. The fitness is calculated as mean square errors between the trained global best weight and the given target obtained by the average weight of the field measurement of the succeed handover transactions.

PSO is originally attributed to Kennedy et al. $[19,20]$ and was first intended for simulating social behavior [21], as a stylized representation of the movement of organisms in a bird flock or fish school. We use the method to first choose which nonlinear neuron to use, and then decide what the state that represents each weight it should be.

The contributions of our work are 1) we are the first to find an important class of the neuron network that combines the memory side with the computation side, and that can mimic the cluster of composite neuron structure like our middle brain; 2) The application of the method to media independent handover for an open-flow control application;

The paper is organized as follows. Section II details for an intercerebral neural network for mobile handover controller and Section III mathematical models for the intercerebral neural network and then shows the simulation results. The last Section is the conclusion and future work.

Modeling the Nonlinear Handover Functions in Intercerebral Neural NETWORK

The underlying nonlinearities from the mobile location to the link parameters and from the link parameters to the final handover decision are complicated. There are interactions among the link parameters of radio signal strength, packet loss, round trip time, available bandwidth, data cost and vehicle speed. Some examples are

- When the radio signal goes down, available bandwidth will go down;

- When the available bandwidth goes down, the packet loss normally goes up then;

- When the packet loss goes up, typically the round trip time will go high as well;

- When the round trip time goes up, the data cost per unit of information may rise up too.

\section{On the other hand, there are cases}

- To keep up with the vehicle speed, the data cost follows up as well;

- When the vehicle velocity speeds up, the signal strength goes down.

In another word, the link parameters are all related to each other through some complicated nonlinear relationships shown later. The only easy way to simplify these relationships is to use the intercelebral neural network that is meant to capture the multiple nonlinear relationships in order for us to make a quick handover decision from the measured field data. By using this method, we are able to capture both WiFi and GPRS characteristics at the same time. The non-linear relationships are first summarized below before we present our PSO (Particle Swarm Optimization) algorithm.

\section{A Mathematical Model of Radio Signal Strength}

The principle of RSSI (Received Signal Strength Indication) ranging describes the relationship between transmitted power and received power of wireless signals with respect to the distance among the nodes. This can be expressed in eqn. (1)

$$
\log (\mathrm{Pr})=\log (\mathrm{Pt})-\mathrm{n}^{\star} \log (\mathrm{d})(1)
$$

Where, $\mathrm{Pr}$ is the received power; $\mathrm{Pt}$ is the transmitted power; $\mathrm{d}$ is the distance between the mobile scanner on the vehicle to the base station or the access point; and $\mathrm{n}$ is the transmission path factor depending on the propagation environment. Note that the typical value $\mathrm{n}$ for $\mathrm{WiFi}$ is around 3 to 4 and for GPRS is around 2 to 3 .

As we can see here, just using one kind of neuron to memorize both values in a media independent mode could be difficult. 


\section{Mathematical Model for Packet Loss}

We cannot obtain a closed formula to describe the packet loss because the queueing system is nonlinear. We can either use numerical calculation or approximation to calculate the packet loss. One approximation is

$$
\log (\mathrm{Pl})=\log (\mathrm{Po})+\mathrm{m}^{\star} \log (\rho)(2)
$$

Where, $\mathrm{Pl}$ is the current packet loss; Po is the initial packet loss, when the system has no buffer to queue the packet; $\rho$ is the current system load condition; and $\mathrm{m}$ is the equivalent buffer size, i.e. the number of average packets that the buffer can hold. Considering the fractal bursty nature of the data traffic, in practical calculation, it could be the number of the biggest packet that the mobile device can handle within the given time limit from the packet source all the way to the final destination. It is again hard to just use homogeneous neurons to capture the buffer sizing situation in an open flow fashion, typical $\mathrm{m}$ for WiFi is bigger than that of GPRS.

\section{Mathematical Model of Round Trip Time}

The principle of Round Trip Time (RTT) ranging [22] describes the relationship between round trip time of wireless signals with respect to the effective distance variation among the nodes. It is shown in formula of (3):

$\operatorname{RTT}(\mathrm{n}+1)=\operatorname{RTT}(\mathrm{n})+\Delta \mathrm{d} / \mathrm{c}(3)$

Where, RTT $(n+1)$ is the RTT for the current packet; RTT(n) is the RTT for the previous packet; and $\mathrm{c}$ is the speed of light. The parameter $\Delta \mathrm{d}$ is the effective distance variation between the mobile on the vehicle and the base station (or the access point), and it follows the Extended Gaussian function, shown below:

$$
\mathrm{f}(\mathrm{x}(\mathrm{t}))=\exp \left(-\mathrm{x}^{\mathrm{g}} /(\mathrm{ft})\right) /(\pi \mathrm{ft})^{1 / 2}(4)
$$

Where, $f(x(t))$ is the probability density function for the $\Delta d$. The parameter $f$ is the Fractal dimension of the process, when $g=2$, the process becomes the Gaussian process. The longer the packet travels, the closer it will be to Gaussian process. A typical value for WiFi is less than 2 and for GPRS it is more than 2. Here again just using one kind of neuron to memorize both open flow values could be difficulty.

\section{Mathematical Model for Available Bandwidth}

The available bandwidth is given by the following formula:

$$
\mathrm{B}=\mathrm{C}-\mathrm{O}(5)
$$

Where, $\mathrm{B}$ is available bandwidth; $\mathrm{O}$ is the overhead consumed by the control and management signals or any non-white noise such as the interference; and $\mathrm{C}$ is the total channel capacity limit (measured in bits/second) given by the famous Shannon theory under the strictly white noise assumption. That is

$$
\mathrm{C}=\mathrm{W}^{\star} \log (1+\mathrm{S} / \mathrm{N})(6)
$$

Where, $\mathrm{W}$ is the bandwidth in $\mathrm{Hz} ; \mathrm{S}$ is the signal strength in watts across the bandwidth $\mathrm{W}$ and $\mathrm{N}$ is the noise power in watts across the bandwidth W.

\section{Mathematical Model of Vehicle Speed}

The model for vehicle speed is simple, and given by

$\mathrm{fd}=\mathrm{V} / \lambda(7)$
Where, $\mathrm{fd}$ is the Doppler frequency spread; $\mathrm{V}$ is the vehicular speed; and $\lambda$ is the wireless wave length. In practice, the mobility model is divided into three categories: a) School or Residential area where the normal speed is 20 miles/hour; b) Urban or Business area where the typical speed is 50 miles/hour; and c) Highway or Rural area where the speed is $80 \mathrm{miles} /$ hour. Take highway as an example, the value for our dual mode mobile scanner is $106 \mathrm{~Hz}$ at $900 \mathrm{MHz}$ GPRS, and $212 \mathrm{~Hz}$ at $1800 \mathrm{MHz}$. At $2.4 \mathrm{GHz} \mathrm{WiFi}$, it is $283 \mathrm{~Hz}$. One can see that using only one type of neuron to memorize all the open flow values at different speed for different RF frequency could be difficult.

\section{Mathematical Model for Data Cost}

There are not so many formula for data pricing for the current GPRS network. Operators normally charge usage by the connection time in seconds. For WiFi network, operators usually charge usage by the total volume in bytes. Either way, the pricing strategy mainly follows the well know Pareto distribution:

\section{$\mathrm{Pc}=(\mathrm{Xm} / \mathrm{x})^{\alpha}(8)$}

Pc is probability of the cost of the service, where $\mathrm{Xm}$ is the minimum possible value of $\mathrm{x}$, representing either connection time or volume, and $\alpha$ is a parameter with a positive value. The Pareto Type I distribution is characterized by a scale parameter Xm and a shape parameter $\alpha$, which is also known as the tail index or the Pareto index. The maximum profit strategy is usually done in such as way that the profits collected from each segmented user groups are relatively equal.

For future cooperative network, the operators may introduce more complicated pricing models (such as charging by the location, by the transmitting power, by the rush hour, or by the hot spot, small cell etc.) in order to maximize the profit. Since the relationship between the cost and the location is nonlinear; using the nonlinear neuron network to guide the open flow handover is always beneficial.

\section{Particle Swarm Optimized INN for Decision Weight Identification}

In an Artificial Neural Network, simple artificial nodes, known as "neurons", "processing elements" or "nonlinear units", are connected together to form a network which mimics a biological neural network.

There is no single formal definition of what an artificial neural network is. However, a class of statistical models may commonly be called "Neural" if they possess the following characteristics:

1. Consist of sets of adaptive linear weights, i.e. numerical parameters that are tuned by a learning algorithm, such as PSO, and

2. Capable of approximating non-linear functions of their inputs, using different nonlinear functions, such as RBF or SBF.

The adaptive weights are conceptually connection strengths between neurons, which are activated during training process, such as PSO.

Particle Swarm Optimization (PSO) is a computational method that optimizes a problem by iteratively trying to improve a candidate solution with regard to a given measure of quality. PSO optimizes a problem by having a population of candidate solutions, here dubbed particles, and moving these particles around in the search-space according to simple mathematical formulae over the particle's position and velocity. Each particle's movement is influenced by its local best known position but, is also guided toward the best known positions in 
Citation: Wu Q, Huang JS, Yang O (2014) An Open Flow Controller Based on the Intercerebral Neural Network for Media Independent Handover. Int J of Swarm Intel Evol Comput 3: 112. doi:10.4303/2090-4908.1000112

Page 5 of 8

the search-space, which are updated as better positions are found by other particles. This is expected to move the swarm toward the best solutions. PSO is originally attributed to Kennedy et al. [19].

The PSO trained INN decision model is shown as Figure 2. Where Logsig is the Sigmoid Based Function (SBF) neuron, and $\operatorname{Exp}(\operatorname{Abs}())$ is our Radial Based Function (RBF) neuron, yt is the vector contains 6 weights listed in Table 2. During the course of computer simulation the mobile parameters are as follows: QVGA display, 2 Mbit pixel camera, one bar code per second, $512 \mathrm{Mb}$ memory; the average driving speed is $50 \mathrm{~km} / \mathrm{hr}$, one trip per day, from Monday to Friday, total five trips. The sample data for learning is measured from the service truck made by the M2M technology Ltd (a subsidiary of GenieView Inc) for Jufeng Group. All the data parameters are normalized for ease of Matlab simulations, i.e. the trained input and output sample data is normalized to accelerate the convergence of neural network optimization process. This neural network consists of one hidden layer, including 6 neurons.

All of the hidden layer neurons adopt either traditional sigmoid or advanced radial based function. This neural network has only one output vector scale, contains the 6 decision weights. Input and output layer neurons adopt pure linear transformation function. For PSO training, the error between the trained neural network output and the expected value from the field testing with the error variation acts as the fitness function. Particle size is 20 , mutation probability is 0.2 , and both initial position and velocity are of zero. The sum of error square is $0.25 \%$ when the neural network is trained less than 64 steps. It is shown that the optimization process is convergent in the course of computer simulation. Thus a PSO trained neural network module is produced to realize the nonlinear mapping relationship between the measured parameters of mobile in the driving vehicle system.

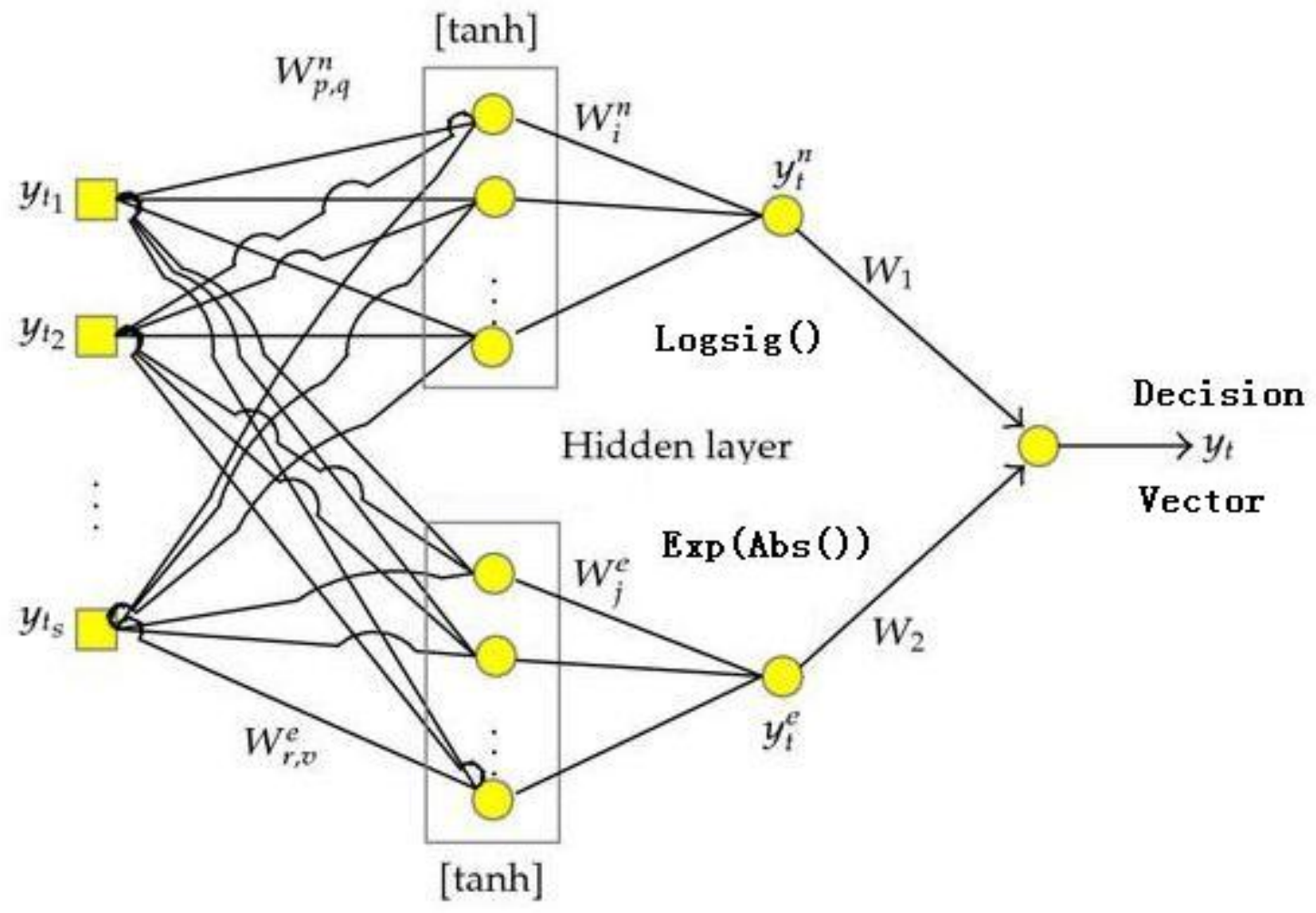

Figure 2: Neural network maps link parameters to handover decision.

The input channel of the trained neural network module is fed with the current link parameters when the system is in motion as shown in Figure 2. The neural network output is produced by the weights trained with computer simulation by the measured field data.

During the training period, PSO algorithm searches for the best solution by making particles moving around the search space according to the global best particle. But when one particle is selected as the global best particle continuously, the other particles may converge at the global best particle repeatedly, which makes the particle swarm fall into local optimization. Especially, for the complex interacting of multiple parameters here, PSO algorithm tends to be premature, so it may not guarantee the convergence to the most advantaged set of INN coefficients.

To overcome the above-mentioned pitfall, the combined algorithm called AMPSO (Particle Swarm Optimization with Adaptive Mutation) is employed as a part of the Genetic Algorithm. Led by nature and the basic principles of physics in a changing environment, AMPSO has the capability to increase population diversity with respect to the genetic variation. It overcomes the problem of premature convergence phenomenon [11], which means the search ends at local minimum, mutation will restarts the search process. To gain more confidence on the INN, we have also trained it by the traditional BP (Backward Propagation). We found that by carefully 
Citation: Wu Q, Huang JS, Yang O (2014) An Open Flow Controller Based on the Intercerebral Neural Network for Media Independent Handover. Int J of Swarm Intel Evol Comput 3: 112. doi:10.4303/2090-4908.1000112

Page 6 of 8

changing the initial conditions, we can end up with the same tables 1 as shown below.

Here is the pseudo code of the algorithm:

1) Initialization

2) Call the fitness calculation subroutine

3) If the error goal is met?

4) No: update the particle position and speed

5) If premature?

6) Yes: mutate the particle, Go back to to 2)

7) No: go back to step 2).
8) Yes: Training ends.

Figures 3-5 show the results of computer simulation for one week of working period. The neural network output tracks the measured output of the controller model closely. The training error is set to less than $0.25 \%$. The simulation is carried out with five days in a cycle; the individual days are plotted on the same graph for easier visual comparison, between different combination of $\log$ sigmoid and absolute exponential radial activations.

The upper sub-graph of figures 3-5 represents the fitness value or training errors, the smaller the better accuracy. The lower sub-graph of them is the actual weight for each link parameters of each day, the more consistent across each day, the better coverage.
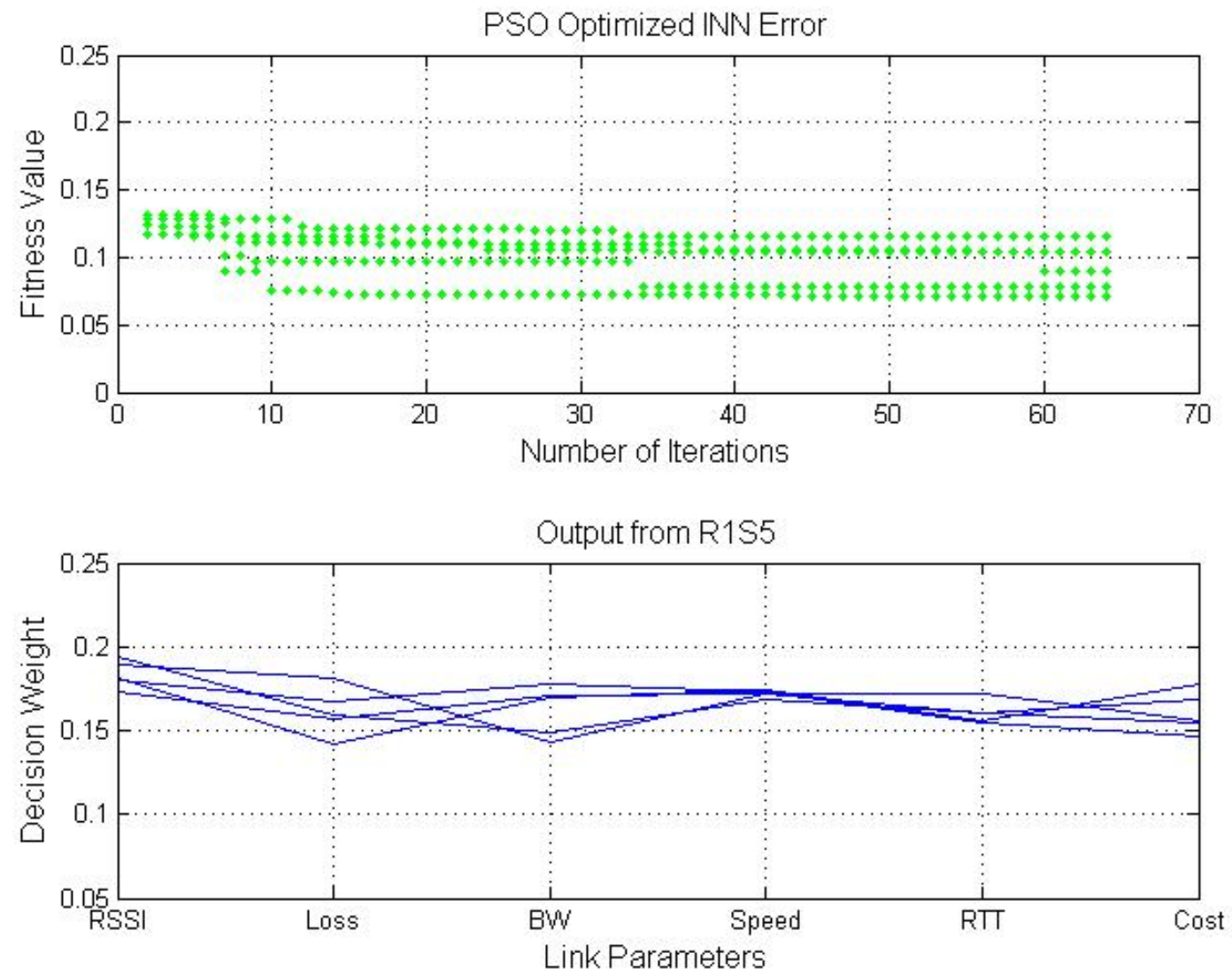

Figure 3: Weight optimized via $1 \mathrm{RBF}$ and $5 \mathrm{SBF}$.

From which, we can see that the Figure 4 presents the best result, where the tracking error is the minimum, and the weights are pretty consistent across the week (covers 5 working days). Figure 6 presents Weight optimized via $3 \mathrm{SBF}$ and $3 \mathrm{RBF}$. 
Citation: Wu Q, Huang JS, Yang O (2014) An Open Flow Controller Based on the Intercerebral Neural Network for Media Independent Handover. Int J of Swarm Intel Evol Comput 3: 112. doi:10.4303/2090-4908.1000112

Page 7 of 8
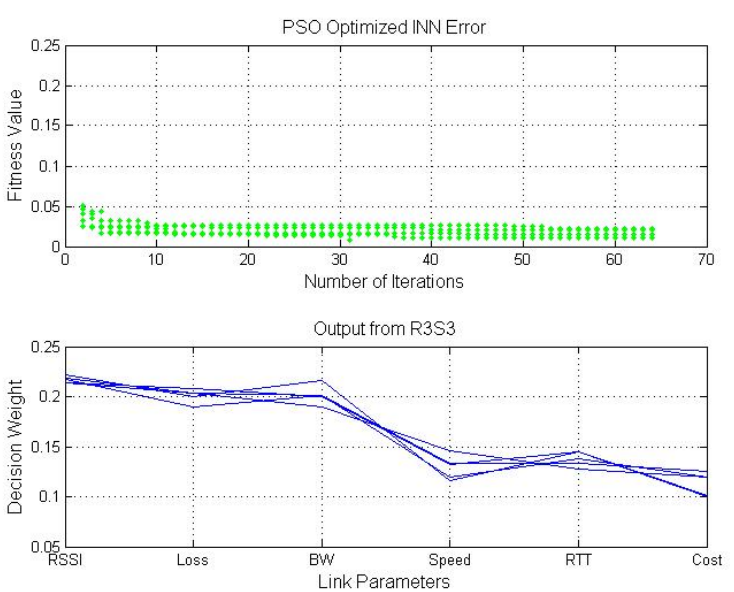

Figure 4: Weight optimized via $3 \mathrm{RBF}$ and $3 \mathrm{SBF}$.
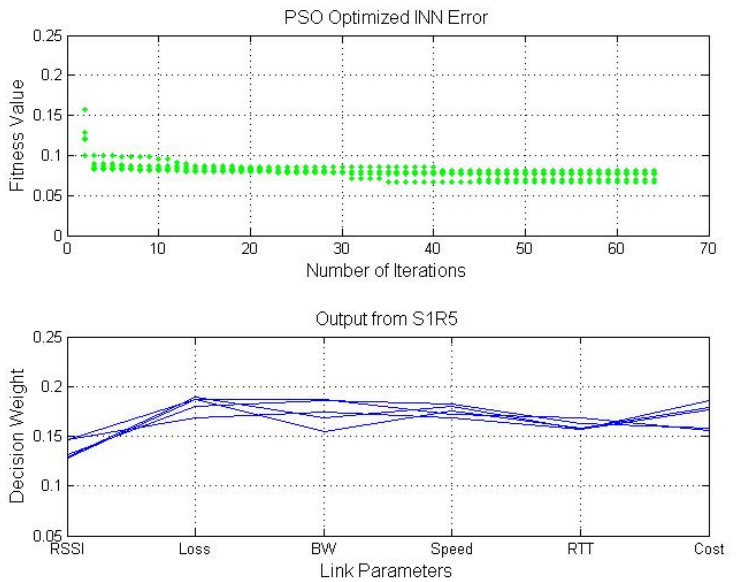

Figure 5: Weight optimized via $1 \mathrm{SBF}$ and $5 \mathrm{RBF}$.
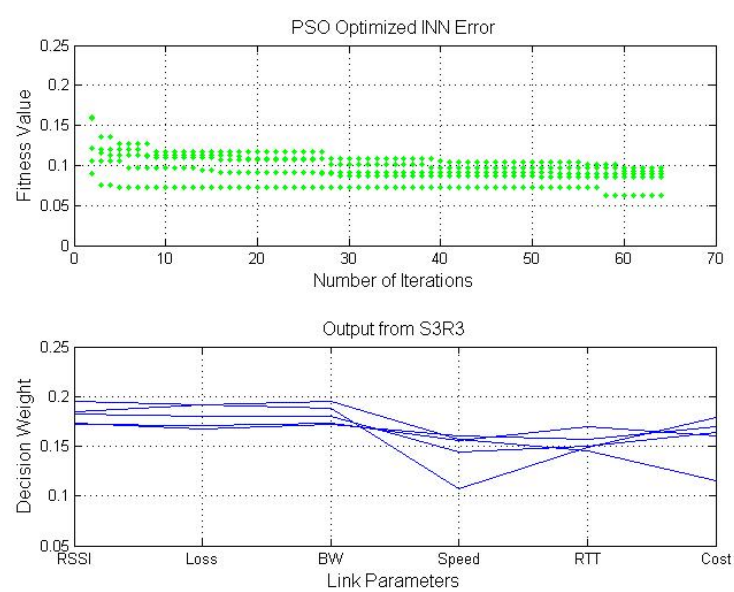

Figure 6: Weight optimized via $3 \mathrm{SBF}$ and $3 \mathrm{RB}$.

Tables 1 and 2 compare the performance of different combinations of RBF and SBF neurons in the hidden layer. Let RxSy to represent the case of using $x$ RBF neurons followed by $y$ SBF neurons in the hidden layer. One can see that the case R3S3 has the smallest weighting error of only $1.5 \%$ indicating that it can converge closer to the theoretical value than the other cases. This is very important observation for the implementation of the algorithm for a number of semi random trips where we would like to a robust neuron structure that can fit all situations. R3S3 is such good candidate because the training error is low, and the trained weight is consistent.

\begin{tabular}{|l|l|l|l|l|}
\hline Merits Optimized & R1S5 & R3S3 & S1R5 & S3R3 \\
\hline Final Error & 0.09 & 0.015 & 0.074 & 0.08 \\
\hline Daily Deviation & 0.02 & 0.005 & 0.008 & 0.017 \\
\hline
\end{tabular}

Table 1: HBF Error with Different Combination.

From above table, we can see the best fit case is six times better than the worst fit case in terms of the accuracy; and the best fit case is four times better than the worst case in terms of the daily standard deviation.

\begin{tabular}{|l|l|l|l|l|l|l|}
\hline $\begin{array}{l}\text { Structure } \\
\text { Weights }\end{array}$ & RSSI & Loss & BW & Speed & RTT & Cost \\
\hline R1S5 & 0.18 & 0.16 & 0.17 & 0.17 & 0.16 & 0.16 \\
\hline R3S3 & 0.22 & 0.2 & 0.19 & 0.14 & 0.13 & 0.12 \\
\hline S1R5 & 0.15 & 0.18 & 0.17 & 0.17 & 0.16 & 0.17 \\
\hline S3R3 & 0.19 & 0.18 & 0.18 & 0.14 & 0.16 & 0.15 \\
\hline Field & 0.21 & 0.19 & 0.18 & 0.15 & 0.14 & 0.13 \\
\hline
\end{tabular}

Table 2: HBF Application with Different Combination.

From above results, we see that the intercerebral method offers the additional flexibility to fit the complicated control of handover situations. Due to its extra nonlinear decision regions, it allows the 
Citation: Wu Q, Huang JS, Yang O (2014) An Open Flow Controller Based on the Intercerebral Neural Network for Media Independent Handover. Int J of Swarm Intel Evol Comput 3: 112. doi:10.4303/2090-4908.1000112

Page 8 of 8

search to be carried out in the WiFi domain and GPRS domain in parallel, while single function can only fit either one at a time, not both.

\section{Conclusions}

The eight equations of a media independent handover for fast delivery logistical vehicle information system that is controlled by open flow controller, is obtained in geographical coordinates system, that takes vehicle speed, route etc into considerations. An intercerebral neural network trained by particle swarm optimization with genetically mutated algorithm identifies the link going down indicate from radio signal strength, packet loss, round trip time, available bandwidth, data cost, vehicle speed based on field test data. The simulation shows that the proposed approach is a viable engineering solution towards the low cost high volume and precise controlling of the media independent handover system. New algorithm makes the trained decision more consistent with each other; in other words, it minimizes the manufacture cost of each smart scanner goes with dispatching vehicle. The only disadvantage is that the initial amount of statistics needed from the field test could be huge, to reach certain level of accuracy; in the moment, the statistics came from the limited routes road test, stored on the cloud servers during service trail period (www.vip56.cn). This paper is to establish a proof of concept and baseline for handover control, by intercerebral neuron methodology. Our simulation indicates that the amount of user satisfaction in terms of daily deviation can be improved is around $37 \%$.

\section{References}

1. Dai S, Zhuang P, Xiang W (2013) GSO: An Improved PSO Based on Geese Flight Theory. Advances in Swarm Intelligence, Harbin, China.

2. Balasubramanian N, Balasubramanian A, Venkataramani A (2009) Energy consumption in mobile phones: a measurement study and implications for network applications. Proceedings of the 9th ACM SIGCOMM conference on Internet measurement. ACM, New York, USA.

3. Li C S, Liao W (2013) Software defined networks. J Comm Mag, IEEE 51: 113-113.

4. Goth G (2011) Software-Defined Networking Could Shake Up More than Packets. J Internet Comput, IEEE 15: 6-9.

5. Taniuchi K, Ohba Y, Fajardo V (2009) IEEE 802.21: Media independen handover: Features, applicability, and realization; J Comm Mag, IEEE 47 112-120.
6. Taylor K, Jadeja JC (2005) Computer aided design and manufacturing: analysis and development of research issues. International Society for Optics and Photonics: 59990Z-12.

7. Cus F, Zuperl U, Kiker E (2006) Adaptive controller design for feedrate maximization of machining process. J Achiev Mat Man Eng 17: 1-2.

8. Yousaf M, Bhatti S, Rehan M (2009) An intelligent prediction model for generating LGD trigger of IEEE 802.21 MIH. Magazine of Emerging Intelligent Computing Technology and Applications. Springer Berlin Heidelberg.

9. Oh S, Hori Y (2007) Parameter Optimization for NC Machine Tool Based on Golden Section Search Driven PSO. IEEE International Symposium on Industrial Electronics, Tokyo.

10. Das Subir, Oba Yoshihiro. MIH pre-authentication: U.S. Patent 8,036,176. 2011-10-11.

11. Jana ND, Tapas Si, Jaya Sil (2012) Particle Swarm Optimization with Adaptive Mutation in Local Bestof Particles. 2012 International Congress on Informatics, Environment, Energy and Applications-IEEA, USA.

12. Jinkun L (2013) Radial Basis Function (RBF) Neural Network Control for Mechanical Systems. Springer.

13. Neruda R, Kudova $P$ (2005) Learning methods for radial basis function networks. Fut Gen Com Learn Sys App 21: 1131-1142.

14. Broomhead DS, Lowe D (1988) Radial basis functions, multi-variable functional interpolation and adaptive networks. Royal Signals And Radar Establishment Malvern (United Kingdom) 3: 321-355.

15. Ho D, Boyes K, Donohoo S (2004) Numerical flow analysis for spillways. Ancold Bulletin, 4: 127-138.

16. Chatila J, Tabbara M (2004) Computational modeling of flow over an ogee spillway. Computers \& structures 82: 1805-1812.

17. Anticevic A, Cole MW, Repovs G, Savic A, Naomi R et al. (2013) Connectivity, pharmacology, and computation: toward a mechanistic understanding of neural system dysfunction in schizophrenia, Frontiers in Psychiatry.

18. Biswal BB, Mennes M, Zuo XN (2010) Toward Discovery Science of Human Brain Function. Proceedings of the National Academy of Sciences 107: 4734-4739.

19. Shi Y, Eberhart RC (1998) Parameter selection in particle swarm optimization. Evolutionary Programming VII. Springer Berlin Heidelberg, New York, USA

20. Bratton D, Kennedy J (2007) Defining a standard for particle swarm optimization. Swarm Intelligence Symposium, SIS IEEE: 120-127, Honolulu, HI.

21. Pedersen $\mathrm{MEH}$, Chipperfield AJ (2010) Simplifying particle swarm optimization. App Soft Comp 10: 618-628.

22. Renker G , Fairhurst G (2011) Sender RTT Estimate Option for the Datagram Congestion Control Protocol (DCCP) IETF RFC 6323. 